\title{
BmmimOAc-Catalyzed Direct Condensation of 2-(Arylamino) Alcohols to Synthesize 3-Arylthiazolidine-2-thiones
}

\author{
CHEN Bihua ${ }^{1, \dagger}$, ELAGEED Elnazeer H. M. ${ }^{1,2, \dagger}$, ZHANG Yongya ${ }^{1}$, GAO Guohua ${ }^{1, *}$ \\ ${ }^{1}$ Shanghai Key Laboratory of Green Chemistry and Chemical Processes, School of Chemistry and Molecular Engineering, \\ East China Normal University, Shanghai 200062, P. R. China. \\ ${ }^{2}$ Department of Chemistry, Faculty of Education, University of Khartoum, Umdurman 406, Sudan.
}

\begin{abstract}
Thiazolidine-2-thiones have attracted much attention because of their unique bioactivity and have been widely used in the fields of medicine and synthetic heterocyclic chemistry. In this work, a simple and convenient route for the synthesis of 3-arylthiazolidine-2thiones by direct condensation of 2-(arylamino) alcohols

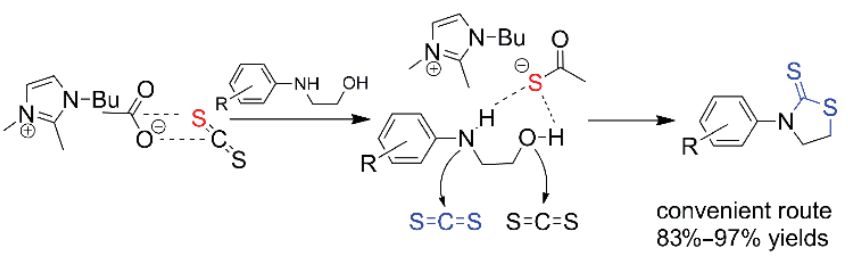
with carbon disulfide $\left(\mathrm{CS}_{2}\right)$ catalyzed by the ionic liquid 1butyl-2,3-dimethylimidazolium acetate (BmmimOAc) has been developed. A series of ionic liquids were used as catalysts in the model reaction of 2-(phenylamino) ethanol with $\mathrm{CS}_{2}$. The results showed that only the acetate ionic liquids have catalytic activity, perhaps owing to the basicity of the acetate anion. Among these acetate ionic liquids, BmmimOAc showed the highest catalytic activity and was selected as the best catalyst. The effects of reaction time, reaction temperature, amount of BmmimOAc, and $\mathrm{CS}_{2}$ to 2-(phenylamino) ethanol molar ratio were investigated in detail. The following were found to be the optimal reaction conditions for direct condensation of 2-(phenylamino) ethanol with $\mathrm{CS}_{2}$ : reaction time, $6 \mathrm{~h}$; reaction temperature, $130{ }^{\circ} \mathrm{C} ; 10 \%$ molar fraction of BmmimOAc; and $\mathrm{CS}_{2}$ to 2-(phenylamino) ethanol molar ratio of $5: 1$. Under these optimized reaction conditions, the product 3-phenylthiazolidine-2-thione was obtained in $97 \%$ yield. The reaction scope was explored by investigating the reactions of various 2-(arylamino) alcohols with $\mathrm{CS}_{2}$. The results showed that the 2-(arylamino) alcohols with electron-donating substituents, electron-withdrawing substituents, or high steric hindrance could be smoothly converted to the corresponding products in excellent isolated yields of $83 \%-95 \%$. NMR characterizations and mass spectrum indicated that the acetate anion of BmmimOAc could react spontaneously with $\mathrm{CS}_{2}$ to form the thioacetate anion $\left(\mathrm{CH}_{3} \mathrm{COS}^{-}\right)$. lonic liquid 1-butyl-2,3-dimethylimidazolium thioacetate (BmmimCOS) might be the actual catalyst in the reaction of 2-(arylamino) alcohols with $\mathrm{CS}_{2} .{ }^{1} \mathrm{H}$ and ${ }^{13} \mathrm{C}$ NMR spectroscopies were used to study the interactions between BmmimCOS and substrates 2-(phenylamino) ethanol and $\mathrm{CS}_{2}$. The NMR spectra showed the hydrogen bonding interactions between BmmimCOS and 2-(phenylamino) ethanol. The $\mathrm{CH}_{3} \mathrm{COS}^{-}$anion of $\mathrm{BmmimCOS}$ could activate the 2-(phenylamino) ethanol in the catalytic system. Based on the characterization results, a possible reaction mechanism was proposed. Firstly, BmmimOAc reacted spontaneously with $\mathrm{CS}_{2}$ to form BmmimCOS. Then, $\mathrm{CH}_{3} \mathrm{COS}^{-}$of BmmimCOS activated 2-(phenylamino) ethanol via hydrogen bonding. Subsequently, $\mathrm{CS}_{2}$ reacted with the activated 2-(phenylamino) ethanol to form the intermediate. Finally, the intermediate was subjected to intramolecular cyclization to form the final product 3-phenylthiazolidine-2-thione.
\end{abstract}

Key Words: Ionic liquids; Homogeneous catalysis; Reaction mechanism; Condensation; 3-Arylthiazolidine-2thiones

\footnotetext{
Received: January 29, 2018; Revised: March 5, 2018; Accepted: March 5, 2018; Published online: March 8, 2018.

${ }^{\dagger}$ Authors contributed equally to this work.

*Corresponding author. Email: ghgao@chem.ecnu.edu.cn; Tel.: +86-21-62233323.

The project was supported by the National Key Research and Development Program of China (2017YFA0403102), National Natural Science Foundation of China (21773068, 21573072), and Shanghai Leading Academic Discipline Project, China (B409).

国家重点研发计划(2017YFA0403102), 国家自然科学基金资助项目(21773068, 21573072)和上海市重点学科建设项目(B409)

(C) Editorial office of Acta Physico-Chimica Sinica
} 


\title{
离子液体 BmmimOAc 催化 2-芳氨基乙醇与二硫化碳一步缩合合成 3-芳基-2-噻唑硫酮
}

\author{
陈必华 ${ }^{1, \dagger}$ ，ELAGEED Elnazeer H. M. ${ }^{1,2, \dagger}$ ，张永亚 ${ }^{1}$ ，高国华 ${ }^{1,}{ }^{*}$ \\ 1 华东师范大学化学与分子工程学院, 上海市绿色化学与化工过程绿色化重点实验室, 上海 200062 \\ 2 喀土穆大学教育学院化学系, 思图曼 406, 苏丹
}

\begin{abstract}
摘要: 噻唑硫酮因具有独特的生物活性, 使其在医学和杂环化学等领域有着广泛的应用, 从而引起了科研工作者的研究 兴趣。本文以离子液体1-丁基-2,3-二甲基咪唑鎓醋酸盐(BmmimOAc)为催化剂, 2-芳氨基乙醇和二硫化碳为起始原料, 一步缩合合成3-芳基-2-噻唑硫酮。以2-苯氨基乙醇和二硫化碳的反应为模型, 考察了一系列离子液体的催化活性。发现 只有阴离子为醋酸根的离子液体才具有催化活性, 这可能是由醋酸根的碱性所导致的。在这些阴离子为醋酸根的离子液 体中, BmmimOAc的催化活性最高。以其为催化剂, 系统考察了反应时间、反应温度、催化剂用量以及二硫化碳和2-苯 氨基乙醇摩尔比对该反应的影响。得到最优的反应条件：反应时间 $6 \mathrm{~h}$ 、反应温度 $130{ }^{\circ} \mathrm{C} 、 10 \%$ 的 $\mathrm{BmmimOAc}$ 用量以及 $5: 1$ 的二硫化碳和2-苯氨基乙醇摩尔比。在该反应条件下，目标产物3-苯基-2-噻唑硫酮的收率达到了97\%。以不同的2芳氨基乙醇为原料, 考察了该反应的普适性。结果表明无论是具有给电子基团、吸电子基团或较大空间位阻的2-芳氨基 乙醇均可顺利地与二硫化碳反应生成相应的3-芳基-2-噻唑硫酮，且分离收率高达 $83 \%-95 \%$ 。核磁共振波谱和质谱分析 表明反应过程中BmmimOAc的醋酸根阴离子可以自发地与二硫化碳反应生成硫代醋酸根阴离子, 因此离子液体1-丁基2,3-二甲基咪唑鎓硫代醋酸盐(BmmimCOS)可能是2-芳氨基乙醇和二硫化碳反应的催化剂。通过核磁共振波谱研究了 BmmimCOS与反应底物2-苯氨基乙醇和二硫化碳之间的相互作用, 发现BmmimCOS与2-苯氨基乙醇之间存在氢键相互 作用。在反应过程中硫代醋酸根阴离子通过氢键作用活化2-苯氨基乙醇, 从而促进反应高效进行。基于表征结果, 提出 了一个可能的反应机理。首先, BmmimOAc自发地与二硫化碳反应生成BmmimCOS。然后, BmmimCOS中的硫代醋酸 根阴离子通过氢键作用活化2-苯氨基乙醇。随后, 活化的2-苯氨基乙醇与二硫化碳反应生成中间体。最后, 中间体分子 内环化生成目标产物3-苯基-2-噻唑硫酮。
\end{abstract}

关键词：离子液体；均相催化；反应机理；缩合反应；3-芳基-2-噻唑硫酮 中图分类号: 0643

\section{Introduction}

Thiazolidine-2-thiones are important heterocyclic compounds in the field of medicine and synthetic organic chemistry. They have attracted much attention due to their unique bioactivity and have been used as antifungal, antimicrobial, antitumoral, antiparasitic, antithyroid and fluorescent-labeling reagents ${ }^{1-7}$. Besides, they also have been used as selective acylating agents ${ }^{8}$, chiral auxiliaries ${ }^{9}$, and ligands in coordination chemistry ${ }^{10,11}$. Therefore, many strategies have been developed for the synthesis of thiazolidine-2-thiones, including the cycloaddition of aziridines with carbon disulfide $\left(\mathrm{CS}_{2}\right)^{12,13}$, the iodocyclization of allyl amines, $\mathrm{CS}_{2}$ and iodine ${ }^{14}$, the reaction of propargylamines with $\mathrm{CS}_{2}{ }^{15,16}$, and the reaction of 2-amino ethanol with trithiocarbonates ${ }^{17}$. These approaches have some disadvantages such as the use of toxic or expensive catalysts, hazardous materials, long reaction time, or high reaction temperature. In addition, a simple and effective process for the synthesis of thiazolidine-2-thiones by the reaction of $\beta$-amino alcohols and $\mathrm{CS}_{2}$ have also been reported $2,3,18,19$. And this method always used strong basic medium $\left(\mathrm{NaOH}, \mathrm{KOH}, \mathrm{Et}_{3} \mathrm{~N}\right.$ etc.) as catalyst.
Ionic liquids have been widely used as reaction media and catalysts due to their unique properties, such as negligible vapor pressure, good thermal stability, and excellent solvent power for both organic and inorganic substances ${ }^{20-23}$. Among these, acetate ionic liquids were considered as high efficient catalysts in many organic reactions because of their basicity of acetate anion ${ }^{24}$. During our previous work on the application of acetate ionic liquids or acetate poly(ionic liquid)s as catalysts, we reported some green synthetic approaches for the synthesis of oxazolidinones ${ }^{25-32}$. We found that ionic liquids or poly(ionic liquid)s with acetate anion are highly active catalysts to produce oxazolidinones in good to excellent yields. Herein, we wish to report the catalytic activities of ionic liquids for synthesis of 3arylthiazolidine-2-thiones in high yields by effective, convenient and eco-friendly approach via direct condensation of 2(arylamino) alcohols with $\mathrm{CS}_{2}$ in the presence of catalytic amount of ionic liquid 1-butyl-2,3-dimethylimidazolium acetate (BmmimOAc) (Scheme 1).

\section{Experimental section 2.1 Materials and reagents}




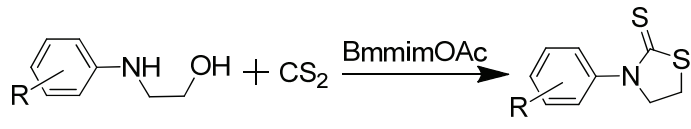

Scheme 1 Synthesis of 3-arylthiazolidine-2-thiones by the reaction of 2-(arylamino) alcohols with $\mathrm{CS}_{2}$ catalyzed by ionic liquid BmmimOAc.

$\mathrm{CS}_{2}$ (AR grade) was supplied by Sinopharm. 2-(Phenylamino) ethanol (>98\% purity) was obtained from TCI. All ionic liquids (>99\% purity) were supplied by Center of Green Chemistry and Catalysis, LICP, CAS. The other reagents were commercial reagents of AR grade supplied by Sinopharm and used without further purification. The 2-(arylamino) alcohols were synthesized by the reaction of arylamines with ethylene carbonate (EC) in the presence of ionic liquid 1-butyl-3methylimidazolium bromide $(\mathrm{BmimBr})$ as catalyst (see Supporting Information)

\subsection{Methods}

GC analysis was performed by using a Shimadzu GC-14B (Shimadzu, Japan) equipped with a capillary column DM-1701 $(30 \mathrm{~m} \times 0.32 \mathrm{~mm} \times 0.25 \mu \mathrm{m})$ and a flame ionization detector. ${ }^{1} \mathrm{H}$ NMR and ${ }^{13} \mathrm{C}$ NMR spectra were recorded at $400 \mathrm{MHz}$ and 100 $\mathrm{MHz}$ on Bruker Ascend 400 spectrometer (Bruker Daltonics Inc., America) with tetramethylsilane as the internal standard. Electrospray ionization mass spectrometry (ESI-MS) analyses was performed on a Bruker MicrOTOF-Q II ESI-MS instrument (Bruker Daltonics Inc., America).

\subsection{Typical procedure for the reaction of 2- (arylamino) alcohols with $\mathbf{C S}_{2}$}

The reaction was carried out in a $15 \mathrm{~mL}$ thick-walled pressure bottle. 2-(Arylamino) alcohols (2.0 mmol), $\mathrm{CS}_{2}(10.0 \mathrm{mmol})$ and BmmimOAc $(10 \%$ molar fraction, $0.2 \mathrm{mmol})$ were mixed together and stirred at $130{ }^{\circ} \mathrm{C}$ for $6 \mathrm{~h}$. The reaction mixture was analyzed by GC with $n$-dodecane as the internal standard. The pure products were obtained by chromatography on silica gel and structurally characterized by NMR spectra.

\section{Results and discussion}

\subsection{Reaction of 2-(phenylamino) ethanol with $\mathrm{CS}_{2}$ catalyzed by various ionic liquids}

Imidazolium-based ionic liquids were proved to be effective catalysts in many organic reactions ${ }^{20,21}$. On this basis, we investigated the reaction of 2-(phenylamino) ethanol with $\mathrm{CS}_{2}$ in the presence of a series of imidazolium-based ionic liquids.

The results were shown in Table 1 . Without any catalyst, no conversion was observed (entry 1), which indicates that the catalyst was required to activate the reaction. Upon addition of a catalytic amount of BmmimOAc as catalyst, 97\% yield of 3phenylthiazolidine-2-thione was obtained (entry 2). However, when using 1-butyl-3-methylimidazolium acetate (BmimOAc), in which the $\mathrm{C}-2$ proton of the imidazolium ring was not replaced by a methyl group, the yield dropped to $68 \%$ (entry 3 ). The reason was that $\mathrm{CS}_{2}$ can react spontaneously with BmimOAc in the liquid phase to form a stable inner-salt compound 1-butyl-3methylimidazolium-2-thiocarboxylate $\left(\mathrm{Bmim}^{+}-\mathrm{COS}^{-}\right)$which
Table 1 Reaction of 2-(phenylamino) ethanol with $\mathrm{CS}_{2}$ catalyzed by various catalysts a.

\begin{tabular}{ccc}
\hline Entry & Catalyst & Yield $/ \%^{\mathrm{b}}$ \\
\hline 1 & none & 0 \\
2 & BmmimOAc & 97 \\
3 & BmimOAc & 68 \\
4 & TBAA & 52 \\
5 & PipOAc & 15 \\
6 & BmmimCl & 0 \\
7 & BmmimBr & 0 \\
8 & BmimCl & 0 \\
9 & BmimBr & 0 \\
10 & BmmimBF 4 & 0 \\
11 & $\mathrm{DBU}^{4}$ & 0 \\
\hline
\end{tabular}

${ }^{\text {a }}$ Reaction conditions: 2-(phenylamino) ethanol: $2 \mathrm{mmol}, \mathrm{CS}_{2}: 10 \mathrm{mmol}$, catalyst: $0.2 \mathrm{mmol}, 130{ }^{\circ} \mathrm{C}, 6 \mathrm{~h} .{ }^{\mathrm{b}} \mathrm{GC}$ yield.

was got by chromatography on silica gel and structurally characterized by NMR spectra (see Supporting Information). Danten and coworkers ${ }^{33,34}$ have reported the same result, and in their studies on chemical reactions of $\mathrm{CS}_{2}$ with ionic liquids, they confirmed that $\mathrm{CS}_{2}$ can undergo degradation to form such compounds. Gao, $\mathrm{Mu}$ and coworkers ${ }^{35}$ also have summarized some reactions of imidazolium-based ionic liquid with inorganic electrophiles (such as $\mathrm{CO}_{2}, \mathrm{CS}_{2}$ and OCS) under alkaline conditions. Meanwhile, by using tetrabutylammonium acetate (TBAA) as catalyst, only 52\% yield of 3-phenylthiazolidine-2thione was obtained (entry 4). This might be due to the weaker alkaline of TBAA compare with BmmimOAc ${ }^{36}$. Moreover, piperidinium acetate (PipOAc) gave the lowest yield of $15 \%$ (entry 5) in these acetate ionic liquids. Besides, due to the lack of active sites, no conversion was detected when using 1-butyl2,3-dimethylimidazolium chloride $(\mathrm{BmmimCl})$, 1-butyl-2,3dimethylimidazolium bromide (BmmimBr), 1-butyl-3methylimidazolium chloride (BmimCl), BmimBr, 1-butyl-2,3dimethylimidazolium tetrafluoroborate $\left(\mathrm{BmmimBF}_{4}\right)$ and $1,8-$ diazabicyclo[5.4.0]undec-7-ene (DBU) as catalyst (entries 611). These results implied that both the acetate anion and 1butyl-2,3-dimethylimidazolium cation were indispensable factors to ensure a good performance in the reaction.

\subsection{Optimization of the reaction parameters}

\subsubsection{The effect of reaction time}

The reaction of 2-(phenylamino) ethanol with $\mathrm{CS}_{2}$ catalyzed by BmmimOAc was carried out in different time range from 1 to $9 \mathrm{~h}$. The effects of reaction time on the yield of 3phenylthiazolidine-2-thione were shown in Fig. 1. From this figure, it can be seen that the reaction affected by the reaction time. The yield of 3-phenylthiazolidine-2-thione was increased by increasing the reaction time till reached $97 \%$ at $6 \mathrm{~h}$, and then the yield remains constant even continued to extend the reaction time.

\subsubsection{The effect of reaction temperature}

The reaction of 2-(phenylamino) ethanol and $\mathrm{CS}_{2}$ was carried out in the temperature range from 100 to $130{ }^{\circ} \mathrm{C}$. The effects of 


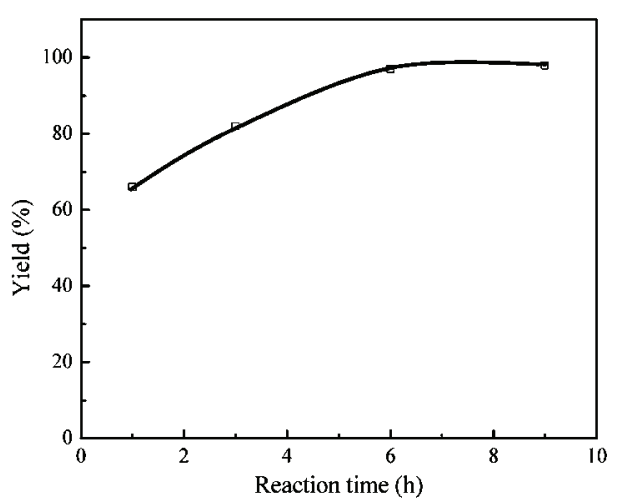

Fig. 1 Effect of reaction time.

Reaction conditions: 2-(phenylamino) ethanol: 2 mmol, $\mathrm{CS}_{2}$ : 10 mmol, BmmimOAc: $0.2 \mathrm{mmol}, 130^{\circ} \mathrm{C}$.

reaction temperature on the yield of 3-phenylthiazolidine-2thione were shown in Fig. 2. It is appears that the reaction was sensitive to the temperature. The yield of 3-phenylthiazolidine2-thione increased smoothly with increase of the temperature from 100 to $120{ }^{\circ} \mathrm{C}$, and then it increased to near $100 \%$ yield when the temperature reached to $130^{\circ} \mathrm{C}$.

\subsubsection{The effect of catalyst amount}

The effect of catalyst amount on the reaction of 2(phenylamino) ethanol and $\mathrm{CS}_{2}$ were shown in Fig. 3. With the increase of the catalyst from $1 \%$ to $10 \%$ molar fraction, the yield of 3-phenylthiazolidine-2-thione increased dramatically. The highest yield $97 \%$ of 3-phenylthiazolidine-2-thione was obtained when using $10 \%$ molar fraction of BmmimOAc as catalyst.

3.2.4 The effect of molar ratio of $\mathrm{CS}_{2}$ to 2-(phenylamino) ethanol

The effect of substrates molar ratio on the reaction of 2(phenylamino) ethanol and $\mathrm{CS}_{2}$ were shown in Fig. 4. It can be clearly seen that, with the increase of the molar ratio of $\mathrm{CS}_{2}$ to 2-(phenylamino) ethanol from $1: 1$ to $5: 1$, the yield of 3phenylthiazolidine-2-thione increased gradually from $75 \%$ to $97 \%$.

Thus, under the optimized reaction conditions $(5: 1$ molar

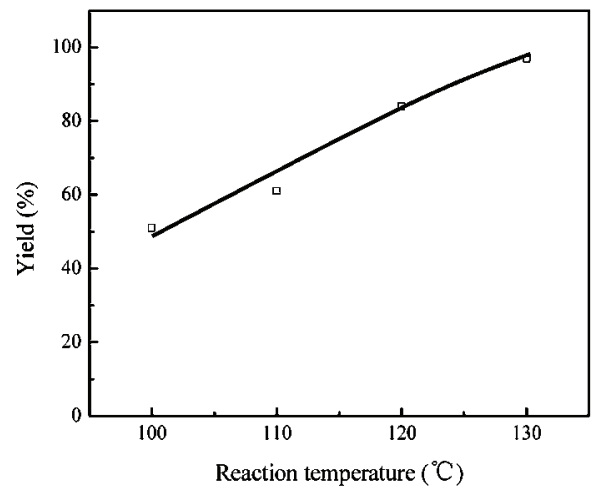

Fig. 2 Effect of reaction temperature.

Reaction conditions: 2-(phenylamino) ethanol, $2 \mathrm{mmol}, \mathrm{CS}_{2}, 10 \mathrm{mmol}$, BmmimOAc, $0.2 \mathrm{mmol} ; 6 \mathrm{~h}$.

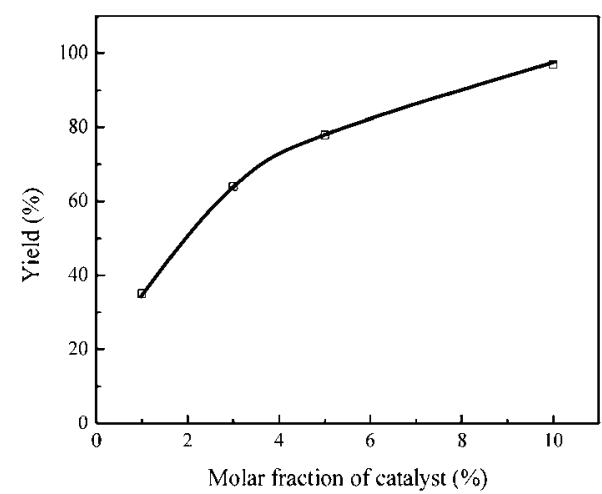

Fig. 3 Effect of catalyst amount.

Reaction conditions: 2-(phenylamino) ethanol, $2 \mathrm{mmol}, \mathrm{CS}_{2}, 10 \mathrm{mmol}, 130^{\circ} \mathrm{C}, 6 \mathrm{~h}$.

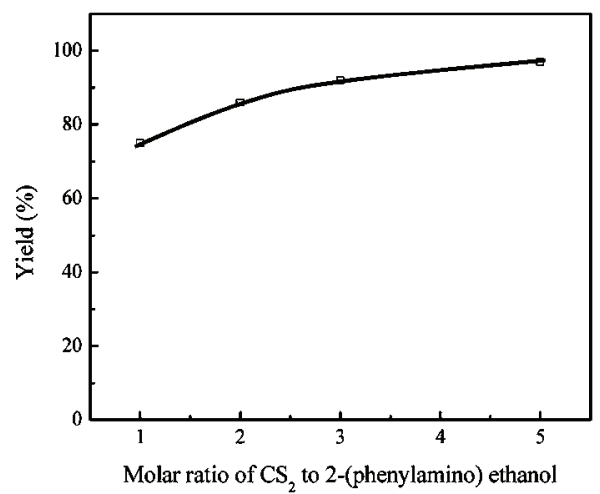

Fig. 4 Effect of molar ratio of $\mathrm{CS}_{2}$ to 2-(phenylamino) ethanol.

Reaction conditions: 2-(phenylamino) ethanol, 2 mmol, BmmimOAc,

$0.2 \mathrm{mmol}, 130^{\circ} \mathrm{C}, 6 \mathrm{~h}$.

ratio of $\mathrm{CS}_{2}$ to 2-(phenylamino) ethanol, $10 \%$ molar fraction of catalyst BmmimOAc, a reaction temperature of $130{ }^{\circ} \mathrm{C}$, and a reaction time of $6 \mathrm{~h}$ ), the highest $97 \%$ yield of 3 phenylthiazolidine-2-thione was obtained in the reaction of 2(phenylamino) ethanol with $\mathrm{CS}_{2}$.

\subsection{Reaction mechanism studies}

In order to investigate the reaction mechanism, ${ }^{1} \mathrm{H}$ NMR and ${ }^{13} \mathrm{C}$ NMR spectroscopies were used to study the interactions between catalyst and substrates. A ${ }^{1} \mathrm{H}$ NMR characterization based on the addition aliquots $\mathrm{CS}_{2}$ to BmmimOAc was carried out. Fig. 5 shows the shifting on ${ }^{1} \mathrm{H}$ NMR spectra of BmmimOAc upon interaction with $\mathrm{CS}_{2}$. When 10 eq. of $\mathrm{CS}_{2}$ was added to the BmmimOAc, the intensity of proton which belong to acetate anion was decreased, accompanied by appearing of a new peak at 2.44. Meanwhile, the proton of imidazolium ring underwent a significant shift from $7.94,7.60$ to $7.65,7.44$ respectively. This might be resulted from the spontaneously reaction of acetate anion with $\mathrm{CS}_{2}$ to form $\mathrm{CH}_{3} \mathrm{COS}^{-}$anion.

For further investigation, ${ }^{13} \mathrm{C}$ NMR characterization based on the addition aliquots $\mathrm{CS}_{2}$ to $\mathrm{BmmimOAc}$ was also carried out. Fig. 6 shows the shifting on ${ }^{13} \mathrm{C}$ NMR spectra of BmmimOAc upon interaction with $\mathrm{CS}_{2}$. When 10 eq. of $\mathrm{CS}_{2}$ was added to BmmimOAc, the $\mathrm{C}=\mathrm{O}$ of acetate anion at 176.51 was disappeared, accompanied by appearing of a new peak at 216.28 . 


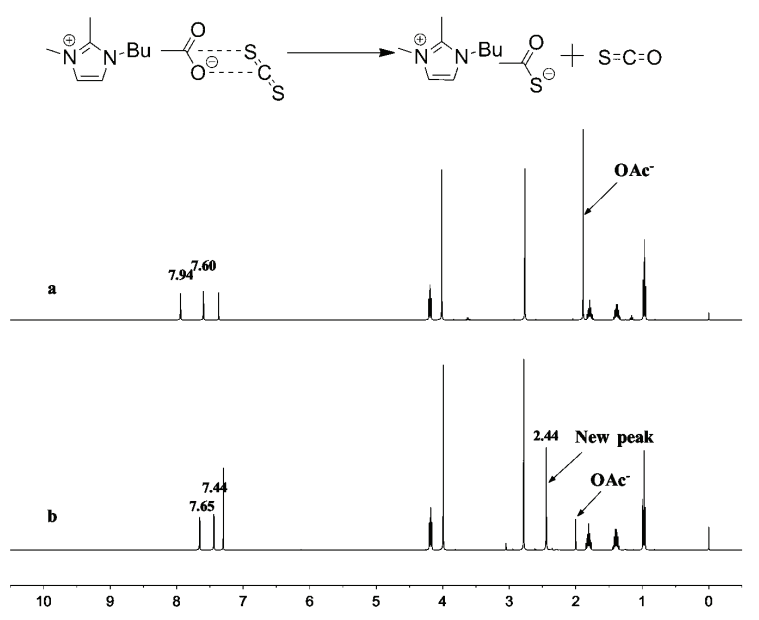

Fig. 5 Chemical shift in the ${ }^{1} \mathrm{H}$ NMR spectra of BmmimOAc.

Solvent: $\mathrm{CDCl}_{3}$. (a) BmmimOAc; (b) BmmimOAc : $\mathrm{CS}_{2}=1: 10$ (molar fraction).

Also the methyl group of acetate anion underwent a significant shift from 25.69 to 39.48 . It suggested that the new peak could be arised from the formation of $\mathrm{CH}_{3} \mathrm{COS}^{-}$anion.

Based on the above NMR characterizations, we did the reaction of BmmimOAc with $\mathrm{CS}_{2}$, and we got the ionic liquid 1butyl-2,3-dimethylimidazolium thioacetate (BmmimCOS) which proved by NMR spectroscopy and mass spectrum (see Supporting Information). The new peaks appeared in Fig. 5 and Fig. 6 completely agreed with the ${ }^{1} \mathrm{H}$ NMR and ${ }^{13} \mathrm{C}$ NMR spectra of BmmimCOS. In addition, Danten and coworkers ${ }^{34,35}$ also have reported that $\mathrm{CS}_{2}$ can react spontaneously with acetate anion of ionic liquid 1-butyl-1-methylpyrrolidinium acetate ([BmPyrro][OAc] $)$ in the liquid phase. All of the results indicated that the acetate anion of BmmimOAc could react spontaneously with $\mathrm{CS}_{2}$ to form $\mathrm{CH}_{3} \mathrm{COS}^{-}$anion. When using the synthesized BmmimCOS as catalyst, 95\% yield of 3phenylthiazolidine-2-thione was obtained in the reaction of 2(phenylamino) ethanol with $\mathrm{CS}_{2}$, which was highly consistent with the result ( $97 \%$ yield) by using BmmimOAc as catalyst. So BmmimCOS might be the actual catalyst in the reaction of 2(arylamino) alcohols with $\mathrm{CS}_{2}$.

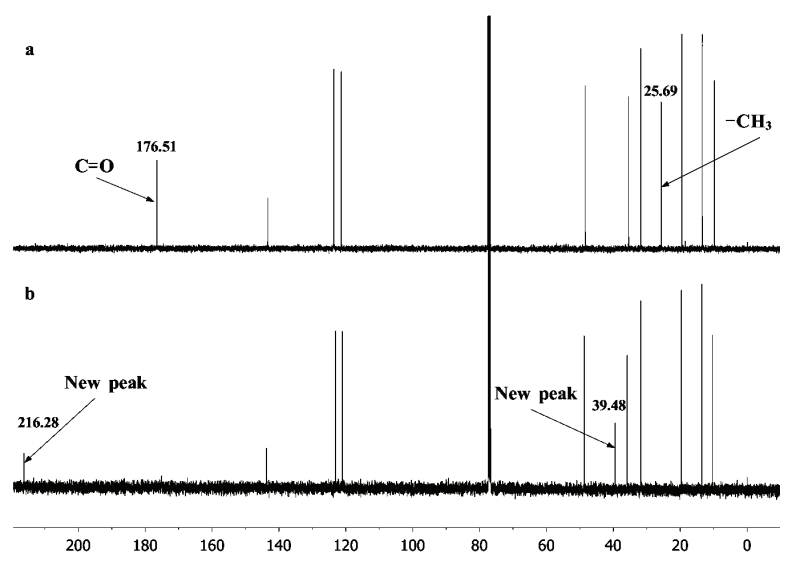

Fig. 6 Chemical shift in the ${ }^{13} \mathrm{C}$ NMR spectra of BmmimOAc. Solvent: $\mathrm{CDCl}_{3}$. (a) BmmimOAc; (b) BmmimOAc : $\mathrm{CS}_{2}=1: 10$ (molar fraction).

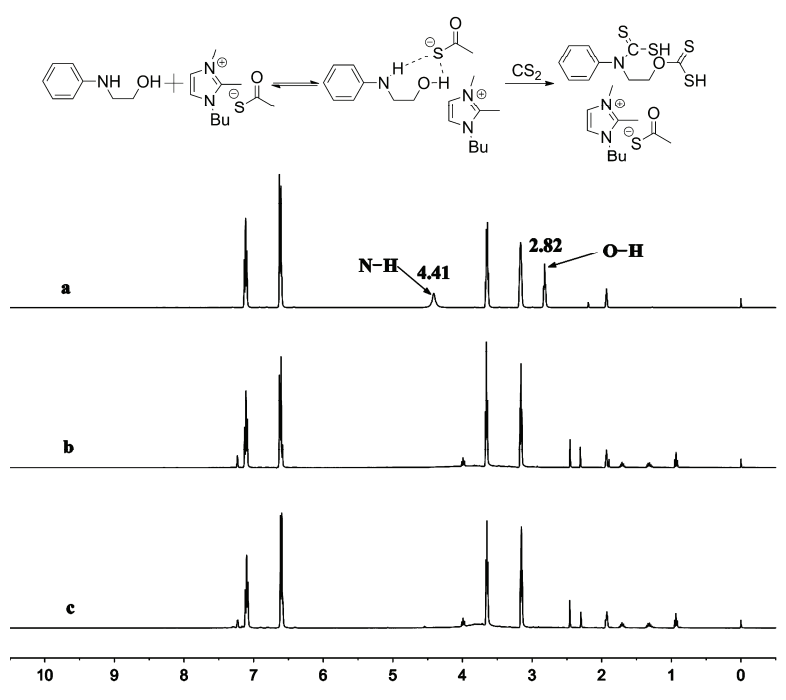

Fig. 7 Chemical shift in the ${ }^{1} \mathrm{H}$ NMR spectra of 2-(phenylamino) ethanol.

Solvent: $\mathrm{CD}_{3} \mathrm{CN}$. (a) 2-(phenylamino) ethanol; (b) BmmimCOS:2-(phenylamino) ethanol $=1: 10 ;$ (c) $\mathrm{CS}_{2}:$ BmmimCOS $: 2$-(phenylamino) ethanol $=50: 1: 10$ (molar fraction).

The interactions between BmmimCOS and substrates were studied by NMR characterizations. Fig. 7 shows the shift on ${ }^{1} \mathrm{H}$ NMR spectra of 2-(phenylamino) ethanol upon interaction with BmmimCOS and $\mathrm{CS}_{2}$. When 0.1 eq. of BmmimCOS was added, the $\mathrm{O}-\mathrm{H}$ proton and the $\mathrm{N}-\mathrm{H}$ proton of 2-(phenylamino) ethanol at 2.82 and 4.41 were disappeared (shown in Fig. 7b). This phenomenon indicated that $\mathrm{CH}_{3} \mathrm{COS}^{-}$anion of BmmimCOS could activate the 2-(phenylamino) ethanol. And this might be resulted from the hydrogen bonding interactions with $\mathrm{CH}_{3} \mathrm{COS}^{-}$anion of BmmimCOS. Meanwhile, upon addition of 5.0 eq. of $\mathrm{CS}_{2}$ to the mixture of 2-(phenylamino) ethanol and BmmimCOS, no obvious change was observed

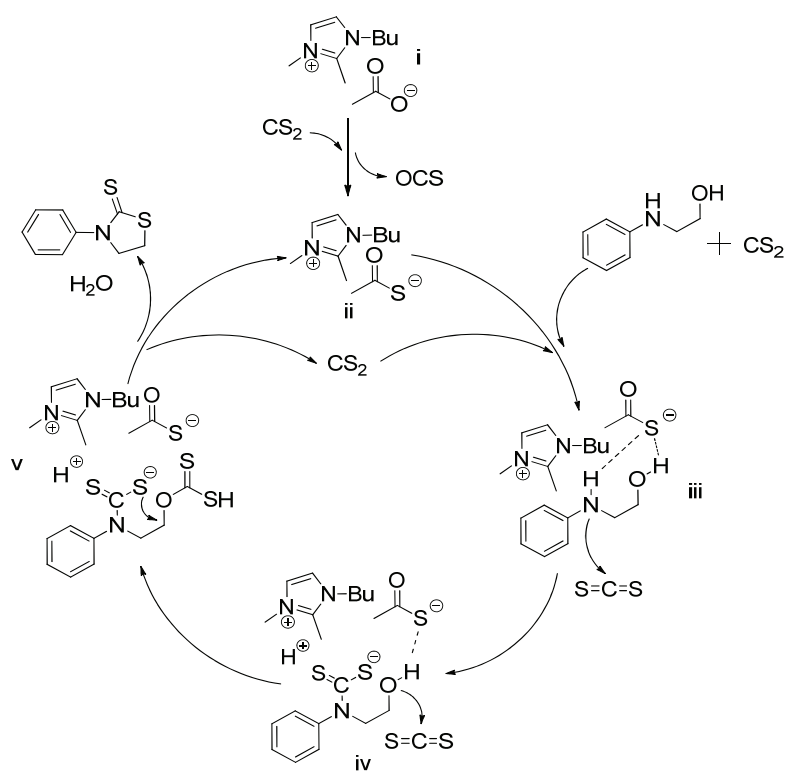

Scheme 2 The proposed reaction mechanism of 2-(phenylamino) ethanol with $\mathrm{CS}_{2}$ catalyzed by ionic liqud BmmimOAc. 
Table 2 Reaction of 2-(arylamino) alcohols with $\mathrm{CS}_{2}$ catalyzed by ionic liquid BmmimOAc. ${ }^{a}$

Entry (-) (Arylamino) alcohols

a Reaction conditions: 2-(arylamino) alcohol, $2 \mathrm{mmol}, \mathrm{CS}_{2}, 10 \mathrm{mmol}$, BmmimOAc, $0.2 \mathrm{mmol}, 130^{\circ} \mathrm{C}, 6 \mathrm{~h}^{\mathrm{b}}{ }^{\mathrm{b}}$ Isolated yield.

(shown in Fig. 7c). In addition, due to the weak interactions of hydrogen bonding between 2-(phenylamino) ethanol and BmmimCOS, no obvious chemical shift was observed in ${ }^{13} \mathrm{C}$ NMR spectra of 2-(phenylamino) ethanol upon addition of 1.0 eq. BmmimCOS and 5.0 eq. $\mathrm{CS}_{2}$ (see Supporting Information). Delaunay and coworkers ${ }^{18}$, Ortiz and Sansinenea ${ }^{37}$ have reported the formation of thiothiazolidines from amino alcohols and $\mathrm{CS}_{2}$, which can be rationalized by the initial formation of the dithiocarbamate, the unhindered primary hydroxyl of dithiocarbamate subsequently attacked to another molecule of $\mathrm{CS}_{2}$ to give the xanthate and the xanthate underwent an intramolecular cyclization to give the thiothiazolidines.

According to the results given by ${ }^{1} \mathrm{H}$ NMR spectra, ${ }^{13} \mathrm{C}$ NMR spectra and mass spectrum, a plausible reaction mechanism for the direct condensation reaction between 2-(phenylamino) ethanol and $\mathrm{CS}_{2}$ was proposed as shown in Scheme 2. Firstly, the acetate anion of BmmimOAc reacted spontaneously with $\mathrm{CS}_{2}$ to form $\mathrm{CH}_{3} \mathrm{COS}^{-}$anion. Then $\mathrm{CH}_{3} \mathrm{COS}^{-}$anion activated 2(phenylamino) ethanol through hydrogen bonding interactions with the hydrogen atom of $\mathrm{O}-\mathrm{H}$ and $\mathrm{N}-\mathrm{H}$ groups. Subsequently, the $\mathrm{CS}_{2}$ reacted with the activated $\mathrm{N}-\mathrm{H}$ group of 2-(phenylamino) ethanol to form dithiocarbamate species iv. Then, the activated $\mathrm{O}-\mathrm{H}$ group of dithiocarbamate species iv attacked to another molecule of $\mathrm{CS}_{2}$ to give the xanthate intermediate v. Finally, the xanthate intermediate $\mathrm{v}$ was subjected to intramolecular cyclization to form final product 3- phenylthiazolidine-2-thione.

\subsection{Reaction of various 2-(arylamino) alcohols with $\mathrm{CS}_{2}$ catalyzed by ionic liquid BmmimOAc}

The scope of the substrates was explored in order to investigate the generalities of this procedure, by the reaction of various 2-(arylamino) alcohols with $\mathrm{CS}_{2}$ in the presence of catalytic amount of BmmimOAc. The results were listed in Table 2. Under the identity optimized reaction conditions, the 2(arylamino) alcohols with either electron-donating substituents (such as $-\mathrm{OMe},-\mathrm{OEt},-\mathrm{Me}$ ) or electron-withdrawing substituents (such as $-\mathrm{Cl},-\mathrm{Br}$ ) were directly converted to the corresponding products in excellent isolated yields from $87 \%$ to 95\% (entries 1-6). Additionally, the high steric hindrance of 2(naphthalen-1-ylamino) ethanol was also applicable for this catalytic system, and it was easily converted to 3-(naphthalen-1yl)-thiazolidine-2-thione in $90 \%$ isolated yield (entry 7 ). Ethanol amine also can smoothly converted to corresponding thiazolidine-2-thione in $83 \%$ isolated yield (entry 8). However, cyclohexylamino alcohol and benzyl amino alcohol which with stronger electronegativity, were relatively inactive (entries 9 and $10)$.

\section{Conclusions}

A simple, convenient and environmentally benign procedure for the synthesis of 3-arylthiazolidine-2-thiones by the condensation reaction of 2-(arylamino) alcohols with carbon disulfide $\left(\mathrm{CS}_{2}\right)$ in the presence of ionic liquid 1-butyl-2,3dimethylimidazolium acetate (BmmimOAc) was provided. Under the optimized reaction conditions, BmmimOAc can catalyze the synthesis of various 3-arylthiazolidine-2-thiones in excellent yields. NMR spectroscopies and mass spectrum indicated that acetate anion of BmmimOAc could react spontaneously with $\mathrm{CS}_{2}$ to form thioacetate anion $\left(\mathrm{CH}_{3} \mathrm{COS}^{-}\right)$, which has a crucial role to activate the substrates through hydrogen bonding interactions. Therefore, the efficient route for synthesis of 3-arylthiazolidine-2-thiones was attributed to the strong hydrogen bond basicity of actual catalyst ionic liquid 1butyl-2,3-dimethylimidazolium thioacetate (BmmimCOS).

Supporting Information: available free of charge via the internet at http://www.whxb.pku.edu.cn.

\section{References}

(1) Prabhakar, Y. S.; Solomon, V. R.; Gupta, M. K.; Katti, S. B. QSAR Studies on Thiazolidines: A Biologically Privileged Scaffold. In QSAR and Molecular Modeling Studies in Heterocyclic Drugs II; Gupta, S. P. Ed.; Springer: Berlin, Germany, 2006; pp. 161-249.

(2) Chen, N.; Du, H.; Liu, W.; Wang, S.; Li, X.; Xu, J. Phosphorus Sulfur Silicon 2015, 190, 112. doi: 10.1080/10426507.2014.931399

(3) Almeida, A. M.; Oliveira, B. A.; Castro, P. P.; Mendonça, C. C.; Furtado, R. A.; Diniz, H. N.; Silva, V. L.; Diniz, C. G.; Tavares, D. 
C.; Silva, H.; et al. Biometals 2017, 30, 841.

doi: 10.1007/s10534-017-0046-6

(4) Li, R.; Ning, X.; Zhou, S.; Lin, Z.; Wu, X.; Chen, H.; Bai, X.; Wang,

X.; Ge, Z.; Li, R.; et al. Eur. J. Med. Chem. 2018, 143, 48. doi: 10.1016/j.ejmech.2017.11.023

(5) Corrêa, R. S.; Silva, M. M.; Graminha, A. E.; Meira, C. S.; Santos, J. A. F.; Moreira, D. R. M.; Soares, M. B. P.; Poelhsitz, G. V.; Castellano, E. E.; Bloch, C.; et al. J. Inorg. Biochem. 2016, 156, 153. doi: 10.1016/j.jinorgbio.2015.12.024

(6) Makiabadi, B. J. Sulfur Chem. 2015, 36, 494. doi: 10.1080/17415993.2015.1062097

(7) Hirata, T.; Kogiso, H.; Morimoto, K.; Miyamoto, S.; Taue, H.; Sano, S.; Muguruma, N.; Ito, S.; Nagao, Y. Bioorg. Med. Chem. 1998, 6, 2179. doi: 10.1016/S0968-0896(98)00156-4

(8) Yamada, S. Tetrahedron Lett. 1992, 33, 2171. doi: 10.1016/0040-4039(92)88169-6

(9) Nagao, Y.; Hagiwara, Y.; Kumagai, T.; Ochiai, M.; Inoue, T.; Hashimoto, K.; Fujita, E. J. Org. Chem. 1986, 51, 2391. doi: $10.1021 /$ jo00362a047

(10) Lobana, T. S.; Rani, A.; Butt, Y. N.; Jasinski, J. P. J. Sulfur Chem. 2015, 36, 251. doi: 10.1080/17415993.2015.1023802

(11) Fabretti, A. C.; Ferrari, M.; Franchini, G. C.; Preti, C.; Tassi, L.; Tosi, G. Transit. Met. Chem. 1982, 7, 279. doi: 10.1007/BF00618715

(12) Clapp, L. B.; Watjen, J. W. J. Am. Chem. Soc. 1953, 75, 1490. doi: $10.1021 / \mathrm{ja} 01102 \mathrm{a} 516$

(13) Sudo, A.; Morioka, Y.; Koizumi, E.; Sanda, F.; Endo, T. Tetrahedron Lett. 2003, 44, 7889. doi: 10.1016/j.tetlet.2003.09.011

(14) Ziyaei-Halimehjani, A.; Marjani, K.; Ashouri, A. Tetrahedron Lett. 2012, 53, 3490. doi: 10.1016/j.tetlet.2012.04.129

(15) Shi, M.; Shen, Y. Heteroatom Chem. 2001, 12, 610. doi: 10.1002/hc.1092

(16) Shen, Y.; Shi, M. Appl. Organometal. Chem. 2003, 17, 767. doi: $10.1002 /$ aoc. 497

(17) Taguchi, T.; Kiyoshima, Y.; Komori, O.; Mori, M. Tetrahedron Lett. 1969, 10, 3631. doi: 10.1016/S0040-4039(01)88473-2

(18) Delaunay, D; Toupet, L.; Corre, M. L. J. Org. Chem. 1995, 60, 6604. doi: 10.1021/jo00125a059

(19) Chen, N.; Jia, W.; Xu, J. X. Eur. J. Org. Chem. 2009, 5841. doi: 10.1002/ejoc.200900759

(20) Welton, T. Chem. Rev. 1999, 99, 2071. doi: 10.1021/cr980032t
(21) Hallett, J. P.; Welton, T. Chem. Rev. 2011, 111, 3508. doi: $10.1021 / \operatorname{cr} 1003248$

(22) Zhang, P.; Wu, T.; Han, B. Adv. Mater. 2014, 26, 6810. doi: 10.1002/adma.201305448

(23) Zhang, Z.; Song, J.; Han, B. Chem. Rev. 2017, 117, 6834. doi: 10.1021/acs.chemrev.6b00457

(24) MacFarlane, D. R.; Pringle, J. M.; Johansson, K. M.; Forsyth, S. A.; Forsyth, M. Chem. Commun. 2006, 1905. doi: 10.1039/B516961P

(25) Zhang, L.; Fu, X.; Gao, G. ChemCatChem 2011, 3, 1359. doi: $10.1002 /$ cctc. 201100016

(26) Wang, B.; Elageed, E. H. M.; Zhang, D.; Yang, S.; Wu, S.; Zhang, G.; Gao, G. ChemCatChem 2014, 6, 278. doi: 10.1002/cctc.201300801

(27) Wang, B.; Yang, S.; Min, L.; Gu, Y.; Zhang, Y.; Wu, X.; Zhang, L.; Elageed, E. H. M.; Wu, S.; Gao, G. Adv. Synth. Catal. 2014, 356, 3125. doi: $10.1002 /$ adsc. 201400026

(28) Elageed, E. H. M.; Wang, B.; Zhang, Y.; Wu, S.; Gao, G. J. Mol. Catal. A: Chem. 2015, 408, 271. doi: 10.1016/j.molcata.2015.07.034

(29) Zhang, L.; Yang, S.; Gao, G. Chin. J. Catal. 2011, 32, 1875. doi: 10.3724/SP.J.1088.2011.10757

(30) Elageed, E. H. M.; Chen, B.; Wang, B.; Zhang, Y.; Wu, S.; Liu, X.; Gao, G. Eur. J. Org. Chem. 2016, 3650. doi: 10.1002/ejoc.201600474

(31) Zhang, Y.; Wang, B.; Elageed, E. H. M.; Qin, L.; Ni, B.; Liu, X.; Gao, G. ACS Macro. Lett. 2016, 5, 435. doi: 10.1021/acsmacrolett.6b00178

(32) Zhang, Y.; Zhang, Y.; Chen, B.; Qin, L.; Gao, G. ChemistrySelect 2017, 2, 9443. doi: 10.1002/slct.201702081

(33) Cabaço, M. I.; Besnard, M.; Chavez, F. V.; Pinaud, N.; Sebastiao, P. J.; Coutinho, J. A. P.; Mascetti, J.; Danten, Y. Chem. Commun. 2013, 49, 11083. doi: 10.1039/c3cc46038j

(34) Cabaço, M. I.; Besnard, M.; Chavez, F. V.; Pinaud, N.;Sebastiao, P. J.; Coutinho, J. A. P.; Danten, Y. J. Chem. Phys. 2014, 140, 244307. doi: $10.1063 / 1.4884820$

(35) Wang, B.; Qin, L.; Mu, T.; Xue, Z.; Gao, G. Chem. Rev. 2017, 117, 7113. doi: 10.1021/acs.chemrev.6b00594

(36) Chen, X.; Song, H.; Chen, P.; Wang, F.; Qian, Y.; Li, X. Acta Chim. Sin. 2012, 70, 770. [陈学伟, 宋红兵, 陈鹏, 王芙蓉, 钱宇, 李雪辉. 化学学报, 2012, 70, 770.] doi: 10.6023/A1108223

(37) Ortiz, A.; Sansinenea, E. J. Sulfur Chem. 2007, 28, 109. doi: $10.1080 / 17415990601139699$ 